\title{
Estudo de fidedignidade e validade da Escala de Avaliação de Dor Psicológica
}

\author{
Liza Fensterseifer \\ Blanca Susana Guevara Werlang ${ }^{1}$
}

\begin{abstract}
Resumo
$\mathrm{Na}$ área da suicidologia, Shneidman propôs a Escala de Avaliação de Dor Psicológica - PPAS. O objetivo deste estudo foi identificar as propriedades psicométricas da PPAS, em adolescentes da população geral, com e sem ideação suicida. A amostra foi de 525 adolescentes, com idade entre 15 e 19 anos, de escolas públicas e privadas de Porto Alegre. Utilizou-se o Inventário de Depressão de Beck, a Escala de Desesperança de Beck, a Escala de Ideação Suicida de Beck e a PPAS. Quanto à fidedignidade, verificou-se um coeficiente de consistência interna de $0,55(p<0,001)$ e de estabilidade temporal de 0,93 ( $p<0,001)$. Quanto à validade discriminante, a PPAS demonstrou ser capaz de diferenciar adolescentes com e sem ideação suicida; para a validade convergente, chegou-se a uma correlação de fraca a moderada; a validade fatorial sugeriu a existência de três dimensões. Concluiu-se que são necessários outros estudos com a PPAS, inclusive com populações clínicas, uma vez que não foram encontradas propriedades psicométricas ideais.

Palavras-Chave: Escala de Avaliação de Dor Psicológica; Fidedignidade; Validade; Adolescentes; Ideação suicida.
\end{abstract}

\section{Study of reliability and validity of Psychological Pain Assessment Scale}

\begin{abstract}
In the suicidology field, Shneidman proposed the Psychological Pain Assessment Scale - PPAS. The objective of this study was identifying the PPAS psychometric properties in adolescents with or without suicide ideation within the general population. The sample included 525 adolescents, aged 15 to 19, attending public and private schools of Porto Alegre, Brazil. Beck Depression Inventory, Beck Scale for Suicidal Ideation and PPAS were used. As to reliability, an internal consistency rate of $0.55(p<0.001)$ was found and, as to temporal stability, the rate was 0.93 $(p<0.001)$. As to discriminant validity, PPAS showed to be able of differentiating adolescents having and not having suicidal ideation; for convergent validity, a weak-to-moderate correlation was found; factorial validity suggested three dimensions. Findings showed further studies would be needed concerning PPAS, including those carried out with clinical populations, as ideal psychometric properties were not assessed.
\end{abstract}

Keywords: Psychological Pain Assessment Scale; Reliability; Validity; Adolescents; Suicide ideation.

\section{Introdução}

Os instrumentos psicológicos precisam passar por procedimentos de avaliação de suas propriedades psicométricas, bem como de atualização. Na resolução no 002/2003, o Conselho Federal de Psicologia determinou que é requisito mínimo e obrigatório para os instrumentos de avaliação psicológica a apresentação de evidências empíricas de validade e fidedignidade.

$\mathrm{Na}$ área da suicidologia, o maior esforço dos estudiosos tem sido a identificação de fatores de risco para o ato suicida, e o desenvolvimento de instrumentos de avaliação auxilia no reconhecimento de grupos de risco, na identificação de sua gravidade e intensidade, e sua associação com diversas variáveis.

Em uma tentativa de entender os comportamentos autodestrutivos e detectar tendências ou predisposições ao ato suicida, Shneidman (1999) propôs a Escala de Avaliação de Dor Psicológica (Psychological
Pain Assessment Scale - PPAS). A PPAS não passou por procedimentos de avaliação de suas propriedades psicométricas, estudo este que Shneidman julga importante que seja realizado, deixando isto a encargo de outros pesquisadores. No manual Diretrizes para o tratamento de transtornos psiquiátricos, editado pela Associação Americana de Psiquiatria e recentemente publicado no Brasil, encontra-se destacado, no capítulo "Avaliação e tratamento de pacientes com comportamento suicida", o uso clínico da PPAS. É mencionado nesta publicação, que este instrumento pode ajudar os profissionais a identificar os mecanismos de enfrentamento e as forças egóicas do paciente, avaliando, igualmente, as necessidades psicológicas que este vê como importantes e insatisfeitas (American Psychiatric Association, 2005).

A PPAS é uma escala em parte subjetiva e em parte objetiva. Por um lado, apresenta-se como um instrumento projetivo temático, semelhante ao TAT (Teste de Apercepção Temática) de Murray, investi-

${ }^{1}$ Endereço para correspondência:

E-mail: bwerlang@pucrs.br 
gando o grau de dor psicológica que o sujeito percebe em cada uma das figuras do instrumento, explorando desta maneira as relações entre dor psicológica aumentada e suicídio. Por outro lado, a PPAS tem uma parte objetiva que se refere aos itens em que o sujeito assinala graus para sua dor psicológica, aponta os sentimentos envolvidos, e manifesta se houve tentativas anteriores de suicídio e o quão letais elas foram.

Mais detalhadamente, a escala consiste em uma folha única de papel, dobrada, de forma a fazer 4 páginas. $\mathrm{Na}$ página 1 encontram-se dados de identificação a serem preenchidos pelo indivíduo que responder ao instrumento, bem como informações conceituais a respeito da dor psicológica. Explicado brevemente o conceito, pede-se que o sujeito classifique sua dor psicológica no momento da testagem, numa escala de 1 a 9, considerando como 1 a menor dor psicológica possível e 9, a maior. Na página 2 e na metade superior da página 3, estão cinco figuras, intituladas Primeiros Passos, A Greve, Partindo para a Guerra, Adão e Eva e Abel Morto e Mulher Pensativa, respectivamente, para as quais o sujeito deve olhar e classificar, em uma escala de 1 a 9, de acordo com o grau de dor psicológica que atribui ao personagem principal da imagem. Em algumas aplicações solicita-se que o sujeito conte uma história sobre cada uma das figuras, ou aponte um título para elas, material que é analisado nos moldes do TAT.

$\mathrm{Na}$ metade inferior da página 3, o sujeito deve classificar a pior dor psicológica que já sentiu, e escolher, em uma listagem de 21 sentimentos, os três que se relacionam a essa dor. Pede-se ainda que manifeste se já tentou o suicídio e, em caso positivo, que indique o método utilizado e o grau de letalidade. Finalmente, na página 4, o sujeito deve descrever como se sentiu em sua pior dor psicológica, em que circunstâncias aconteceu e como esta se resolveu.

Shneidman (1993, 1998, 1999), baseando suas concepções tanto nos elementos motivacionais quanto nos de intencionalidade do ato suicida, bem como na formulação do conceito de dor psicológica, sustenta que o suicídio é causado por uma dor psicológica - em inglês, psychache - insuportável. Dor psicológica refere-se a dor, angústia, e associa-se ao sentimento excessivo de culpa, vergonha, solidão, medo. Mesmo não sendo a solução mais adequada para resolver os problemas, a morte é a saída que o indivíduo encontra para deter o fluxo desta dor insuportável, que tem sua fonte em necessidades psicológicas frustradas (Shneidman, 1998, 1999).

A autodestruição voluntária - o suicídio - é uma ocorrência que tem crescido expressivamente nas últimas décadas (De Leo, Bertolote \& Lester, 2003). Nas estatísticas de causa de morte no mundo, o suicídio aparece entre as dez principais, e para adolescentes com idade entre 15 e 19 anos este número sobe para a terceira colocação. Cabe mencionar que Porto Alegre e Curitiba são apontadas como as capitais brasileiras com maior índice de suicídios registrados entre adolescentes, quando comparadas com outras nove capitais - Belém, Fortaleza, Natal, Recife, Salvador, Belo Horizonte, Vitória, Rio de Janeiro e São Paulo (Souza, Minayo \& Malaquias, 2002). Esses dados demonstram que a prevenção do suicídio é de alta prioridade, configurando um problema de saúde pública.

O comportamento suicida, geralmente, apresentase como um gradiente de gravidade, situando-se a ideação suicida em um dos extremos e o suicídio consumado em outro, ficando a tentativa de suicídio entre eles (Maris, Bermann \& Silverman, 2000). O estudo da ideação suicida é importante, pois é um dos preditores para o risco de suicídio (WHO, 2002).

A adolescência é um período de reorganização psíquica, em que passado e presente precisam ser integrados. Esse processo gera dúvidas, incertezas, conflitos pessoais e familiares, ambivalência e, muitas vezes, um sentimento de dor insuportável, sustentam Leenaars e Lester (1995). O adolescente precisa elaborar lutos e perdas, processo estruturante da personalidade, em um ego com menos recursos defensivos. Eles mencionam ainda que diferentes autores atribuem um caráter suicidógeno aos conflitos típicos da adolescência e, por tudo isso, observa-se que nesta fase podem ser registradas situações de crise, expressas por conflitos, contradições, angústia, violência e comportamentos autodestrutivos. Neste contexto, eventualmente, podem aparecer pensamentos suicidas, que, conforme sua intensidade, profundidade e duração, devem ser considerados ocorrências bastante preocupantes (WHO, 2002).

Existem muitas tentativas de explicar o que motiva uma pessoa a suicidar-se. A idéia de concordância unânime entre os autores é que o suicídio é um evento complexo, multidimensionado, sobre o qual converge uma série de fatores. Neste contexto, mais do que nunca, destaca-se a importância de se ter à disposição instrumentos adequados e confiáveis em relação ao que se quer medir. A PPAS, assim como outros instrumentos que visam à investigação, detecção ou ao entendimento dos atos autodestrutivos, pode ser um valioso auxílio para profissionais da saúde, uma vez que o suicídio, em todas as faixas etárias, e em especial na adolescência, é um fenômeno trágico que deve ser evitado, pois constitui a perda prematura de talentos e recursos que afetam o desenvolvimento de uma sociedade. Este estudo teve, então, como objetivo, identificar as propriedades psicométricas da PPAS em adolescentes da população geral, com e sem ideação suicida.

Psico-USF, v. 10, n. 1, p. 21-29, jan./jun. 2005 


\section{Método}

Para alcançar o objetivo proposto, realizou-se um estudo quantitativo, de tipo transversal. A amostra foi constituída por 525 adolescentes, com idade entre 15 e 19 anos $(M=16,03 ; \mathrm{DP}=1,08)$, sendo 295 (56,2\%) do sexo feminino e 230 (43,8\%) do sexo masculino; 290 $(55,2 \%)$ freqüentavam escolas públicas e 235 (44,8\%), escolas privadas da cidade de Porto Alegre (RS).

Os instrumentos utilizados foram o Inventário de Depressão de Beck (BDI), a Escala de Desesperança de Beck (BHS), a Escala de Ideação Suicida de Beck (BSI) - todos em sua versão brasileira - e a Escala de Avaliação de Dor Psicológica (PPAS).

O BDI (Beck \& Steer, 1993a) é uma escala sintomática de depressão, constituída por 21 itens de múltipla escolha. A soma dos escores dos itens resulta em um escore total, que equivale à intensidade da depressão, que pode ser classificada como mínima, leve, moderada e grave. A versão em português (Cunha, 2001) foi testada em adultos e adolescentes de amostras clínicas e da população geral, apresentando resultados satisfatórios de fidedignidade e validade.

A BHS (Beck \& Steer, 1993b) mede a dimensão do pessimismo ou a extensão das atitudes negativas do indivíduo diante do futuro. É uma escala dicotômica (certo ou errado) que engloba 20 itens. A soma dos itens resulta em um escore total, com base no qual se classifica a desesperança em mínima, leve, moderada e grave. Sua versão em português foi igualmente testada em amostras clínicas e não-clínicas de adolescentes, demonstrando índices adequados de fidedignidade e validade (Cunha, 2001).

A BSI (Beck \& Steer, 1991) é uma medida escalar de auto-relato, que objetiva a identificação da presença de ideação suicida, além de avaliar os planos, comportamentos e atitudes que podem ser encontrados no paciente, para que cometa o suicídio. É constituída por 21 itens, cada um com três alternativas de resposta. Suas propriedades psicométricas, na versão em português (Cunha, 2001), em amostras clínicas e não-clínicas, são consideradas satisfatórias. Especificamente com adolescentes, encontrou-se um coeficiente alfa de Cronbach de 0,93, o que é excelente (Werlang, Borges \& Fensterseifer, 2004).

A PPAS foi originalmente criada nos Estados Unidos, na língua inglesa, o que tornou necessária a sua tradução para o português (Brasil) - trabalho autorizado pelo autor, dr. Edwin Shneidman, em maio de 2002. Para isso, foi escolhido um grupo de peritos (psicólogos clínicos e especialistas em tradução), que atuaram como juízes, para que se chegasse a uma formulação consensual da versão em português, que apresentasse equivalência com a versão inglesa, tanto conceitual quanto semântica.

A equivalência lingüística entre as versões em inglês e em português foi testada após completada a fase de tradução da escala, a partir de sua aplicação em 20 sujeitos bilingües da população geral, com características semelhantes às da amostra deste estudo. O teste e o reteste foram administrados, com variação da ordem de apresentação dos dois idiomas, com dois grupos de 10 sujeitos: 5 começaram respondendo à escala em português e terminaram em inglês, e 5 sujeitos fizeram o contrário. $\mathrm{O}$ intervalo entre teste e reteste do primeiro grupo foi de 3 dias, chegando-se a uma estimativa de correlação de $0,92(p<0,001)$. O segundo grupo realizou o teste e o reteste com 10 dias de intervalo, para que prováveis efeitos relacionados à memória pudessem ser descartados, chegando-se a uma correlação de 0,96 ( $p<0,001)$. Assim, demonstra-se a adequabilidade da tradução da PPAS para a língua portuguesa, que pode ser observada mediante ambos os coeficientes de correlação entre o teste e o reteste.

A versão original da PPAS propõe que o sujeito assinale graus de dor psicológica, por meio de uma medida escalar do tipo Likert, de 1 a 9, objetivando verificar o nível de concordância do sujeito com afirmações favoráveis ou desfavoráveis sobre o objeto psicológico. Geralmente o número de pontos neste tipo de escala de resposta varia de 3 até mais de 10 , sendo as escalas de 5 e 7 pontos as mais utilizadas (Pasquali, 1996). Optou-se, com autorização do autor, por alterar a escala de 1 a 9 , na PPAS original, para 1 a 5 (1 a menor dor psicológica possível, 2 uma dor psicológica baixa ou leve, 3 uma dor psicológica média, 4 uma dor psicológica alta, e 5 uma dor psicológica extremamente alta). Com esta alteração, procurou-se evitar que a amplitude de resultados nas respostas afetasse a consistência interna do instrumento.

Para fins de análise estatística (exceto para verificação da validade discriminante do instrumento) foram utilizados apenas os itens da PPAS passíveis de quantificação, e que tinham sua pontuação através de uma escala Likert (variando de 1 a 5). São eles: intensidade da dor psicológica no momento da aplicação do instrumento (folha 1), intensidade da dor psicológica nas figura 1, 2 e 3 (folha 2), intensidade da dor psicológica nas figura 4 e 5 , intensidade da pior dor psicológica experimentada em toda a vida e grau de letalidade de tentativa prévia de suicídio (folha 3).

Para a coleta de dados realizaram-se contatos com instituições escolares públicas e privadas, e as que colaboraram foram aquelas que acolheram a proposta do estudo. A administração dos instrumentos foi coletiva, na própria escola e em horário escolar.

Para a análise dos dados utilizou-se o SPSS versão 11.5. Em relação às considerações éticas deste estudo, cabe destacar que todos os participantes, assim como os pais ou responsáveis daqueles menores de 18 anos, assinaram o Termo de Consentimento Livre e 
Esclarecido, e que o projeto deste estudo foi aprovado pelo Comitê de Ética em Pesquisa da PUCRS.

\section{Resultados}

Dos 525 adolescentes que participaram da pesquisa, $188(35,8 \%)$ pontuaram presença de ideação suicida na BSI. Destes, 127 eram mulheres e 61 eram homens, conforme consta na Tabela 1. Percebe-se que pouco mais de um terço da amostra tem indicativos, segundo a pontuação obtida na BSI, de ideação suicida.

Tabela 1 - Freqüência e porcentagem de adolescentes com e sem ideação suicida, em relação ao sexo $(n=525)$

\begin{tabular}{lccccccc}
\hline Presença de ideação suicida na BSI & \multicolumn{2}{c}{ Sexo feminino } & \multicolumn{2}{c}{ Sexo masculino } & \multicolumn{2}{c}{ Total } \\
& & $\mathrm{f}$ & $\%$ & $\mathrm{f}$ & $\%$ & $\mathrm{f}$ & $\%$ \\
\hline & Sim & 127 & 43,05 & 61 & 26,52 & 188 & 35,8 \\
& Não & 168 & 56,95 & 169 & 73,48 & 337 & 64,2 \\
\hline Total & 295 & 56,2 & 230 & 43,8 & 525 & 100 \\
\hline
\end{tabular}

\section{Estudo de fidedignidade da PPAS}

\section{Análise de item e consistência interna}

Investigando a consistência interna da PPAS, chegou-se a um coeficiente de 0,55 , o que pode ser considerado de fraco a moderado (Costa Neto, 1983). Os resultados podem ser visualizados na Tabela 2 .
Considera-se importante mencionar que também foi calculado o coeficiente alfa, retirando-se, dentre os itens do instrumento, a figura 4 e a figura 5 , apontadas como aquelas com menor poder de discriminação de pessoas com e sem dor psicológica, chegando-se a coeficientes praticamente iguais.

Tabela 2 - Média, desvio padrão, correlações item e total corrigido e coeficiente de fidedignidade da PPAS $(n=525)$

\begin{tabular}{lcccc}
\hline Variável & $\mathrm{M}$ & $\mathrm{DP}$ & rtot & $\mathrm{p}$ \\
\hline Dor psicológica no momento da aplicação do instrumento & 2,11 & 1,18 & 0,58 & $<0,001$ \\
Dor psicológica na figura 1 & 1,47 & 0,96 & 0,30 & $<0,001$ \\
Dor psicológica na figura 2 & 3,50 & 1,53 & 0,54 & $<0,001$ \\
Dor psicológica na figura 3 & 4,43 & 1,82 & 0,44 & $<0,001$ \\
Dor psicológica na figura 4 & 4,35 & 1,00 & 0,32 & $<0,001$ \\
Dor psicológica na figura 5 & 1,84 & 1,06 & 0,42 & $<0,001$ \\
Pior dor psicológica que o sujeito já teve & 3,67 & 1,25 & 0,60 & $<0,001$ \\
Grau de letalidade da tentativa suicida & 2,18 & 1,21 & 0,59 & $<0,001$ \\
Total & 21,35 & 3,96 & & \\
Fidedignidade & & & 0,55 & \\
\hline
\end{tabular}

\section{Estabilidade temporal}

O estudo da estabilidade temporal da PPAS foi realizado com 39 dos 525 adolescentes que realizaram o teste e o reteste, este sendo aplicado 10 dias após aquele. Utilizando-se o coeficiente de Spearman, a estimativa de correlação foi de $0,93(p<0,001)$, o que pode ser interpretado como sugerindo uma estabilidade temporal satisfatória.

\section{Estudo de validade da PPAS}

\section{Validade discriminante}

Para efeito de análise discriminante, utilizandose os testes não-paramétricos de Mann-Withney e Wilcoxon, comparou-se a pontuação dos adolescentes com e sem ideação suicida. Pelos resultados, concluiu-se que existe diferença estatisticamente significativa entre as médias dos escores obtidos na PPAS, quanto à presença de dor psicológica, pelos dois grupos de adolescentes.

Objetivando, igualmente, investigar o quanto e quais os itens da PPAS (dentre os itens passíveis deste tipo de análise) mais influenciam na classificação do sujeito conforme presença ou ausência de dor psicológica, que neste estudo foi considerada como associada à presença ou ausência de ideação suicida, conforme pontuação na BSI, utilizou-se o modelo de regressão logística. De acordo com o teste de Hosmer e Lemeshow, observando-se o escore de Nagelkerke, concluiu-se que o modelo de regressão logística deve estar adequado, podendo ser considerado confiável $(p=0,062)$.

Inicialmente, verificou-se a influência, observada de forma individual, de cada um dos itens da PPAS, na classificação do adolescente conforme presença ou ausência de ideação suicida (vide Tabela 3). Assim, 
percebeu-se que, conforme o grau de significância das variáveis utilizadas no modelo de regressão, a principal influência na pontuação do adolescente na PPAS, para dor psicológica, se deve, principalmente, aos itens "dor psico- lógica no momento da aplicação do instrumento", "pior dor psicológica experimentada em toda a vida", "tentativas anteriores de suicídio", "método utilizado em tentativas anteriores" e "grau de letalidade de tentativas anteriores".

Tabela 3 - Influência dos itens da PPAS, de forma individual, na classificação do adolescente com e sem dor psicológica

\begin{tabular}{lccc}
\hline Itens da PPAS & Escore & gl & $p$ \\
\hline Dor no momento & 63,82 & 1 & 0,000 \\
Figura 1 & 3,25 & 1 & 0,071 \\
Figura 2 & 7,00 & 1 & 0,008 \\
Figura 3 & 3,62 & 1 & 0,057 \\
Figura 4 & 0,25 & 1 & 0,620 \\
Figura 5 & 2,32 & 1 & 0,128 \\
Pior dor experimentada & 39,00 & 1 & 0,000 \\
Letalidade & 87,65 & 1 & 0,000 \\
Método & 88,36 & 1 & 0,000 \\
Tentativas anteriores & 113,26 & 1 & 0,000 \\
\hline
\end{tabular}

Entretanto, é de interesse verificar a influência das variáveis, de forma conjunta, por meio da regressão logística. Sendo assim, cabe considerar que as variáveis letalidade e métodos utilizados não foram incluídas neste cálculo, tendo em vista que só ocorrem aliadas à existência de tentativas de suicídio. As demais variáveis foram incluídas no modelo, como pode ser observado na Tabela 4. Foram verificadas as suposições do modelo (como multicolinearidade, autocorrelação e homocedasticidade), e estas foram satisfeitas.

Tabela 4 - Influência dos itens da PPAS, de forma conjunta, na classificação do adolescente com e sem dor psicológica

\begin{tabular}{lccc}
\hline Itens da PPAS & Wald & gl & $p$ \\
\hline Dor no momento & 18,20 & 1 & 0,000 \\
Figura 1 & 0,13 & 1 & 0,724 \\
Figura 2 & 2,19 & 1 & 0,139 \\
Figura 3 & 0,34 & 1 & 0,562 \\
Figura 4 & 1,21 & 1 & 0,271 \\
Figura 5 & 0,04 & 1 & 0,840 \\
Pior dor experimentada & 3,99 & 1 & 0,046 \\
Tentativas anteriores & 42,24 & 1 & 0,000 \\
\hline
\end{tabular}

Como pode ser observado, os itens que efetivamente mostraram influência na discriminação de adolescentes com ideação suicida são "dor psicológica no momento da aplicação do instrumento", "pior dor psicológica já experienciada" e "existência de tentativas anteriores de suicídio". Em seu conjunto, as figuras não se mostram suficientes para a determinação da presença ou não de dor psicológica.
Mediante, ainda, o modelo de regressão logística, foi possível observar que a classificação dos adolescentes sem ideação suicida na BSI, pela PPAS, considerando, então, presença ou ausência de dor psicológica, é muito boa (95,5\% de acerto); por outro lado, dentre os adolescentes com ideação suicida na BSI, a capacidade de classificação da PPAS é menor (42\% de acerto). Esses resultados podem ser visualizados na Tabela 5.

Tabela 5 - Classificação dos adolescentes, conforme o observado na BSI e o predito pela PPAS

\begin{tabular}{cccc}
\hline Observado & \multicolumn{2}{c}{ Predito } & \% de acerto \\
\hline Com ideação suicida & 79 & Sem ideação suicida & 42,0 \\
Sem ideação suicida & 15 & 322 & 95,5 \\
\hline Média (\%) & & & 76,4 \\
\hline
\end{tabular}




\section{Validade convergente}

Para verificação da validade convergente da PPAS, o escore total obtido nesta escala foi comparado com o escore total da BSI, o escore total da BHS e o item 9 do BDI, que avalia idéias suicidas. Cruzando-se, então, o escore total da PPAS com o escore total da BSI, chegou-se a uma estimativa de correlação de $r=0,337 \quad(p<0,001)$. O mesmo procedimento foi realizado com o escore total da BHS e com o item 9 do BDI, e verificaram-se relações significantes de $r=0,308$ $(p<0,001)$ e de $r=0,315(\phi<0,001)$, respectivamente.
Todas estas correlações podem ser interpretadas como sendo de fracas a moderadas.

\section{Validade fatorial}

Numa análise fatorial da PPAS, com rotação varimax, foi possível extrair três fatores, com raiz característica maior do que 1 , sugerindo a composição de 3 sub-escalas (vide Tabela 6). Esses fatores correspondem a $60,59 \%$ da variância total explicada. Analisando as cargas fatoriais, obtiveram-se os resultados descritos na Tabela 7.

Tabela 6 - Análise fatorial dos itens da PPAS, raízes características e porcentagem da variância explicada ( $\mathrm{n}=525)$

\begin{tabular}{cccc}
\hline Fator & Raiz característica & \% de variância explicada & \% da variância acumulada \\
\hline 1 & 1,58 & 22,595 & 22,595 \\
2 & 1,45 & 20,764 & 43,359 \\
3 & 1,20 & 17,209 & 60,568 \\
\hline
\end{tabular}

Tabela 7 - Itens que compõem os fatores I, II e III da análise fatorial da PPAS e seus respectivos pesos

\begin{tabular}{ccc}
\hline Fatores & Itens & Peso \\
\hline \multirow{2}{*}{ Fator I } & Figura 2 & 0,730 \\
& Figura 3 & 0,800 \\
Fator II & Figura 4 & 0,616 \\
& Dor psicológica no momento & 0,817 \\
Fator III & Pior dor psicológica & 0,840 \\
& Figura 1 & 0,859 \\
& Figura 5 & 0,421 \\
\hline
\end{tabular}

\section{Discussão dos resultados}

A PPAS foi estudada quanto a sua fidedignidade e validade, na tentativa de definir se ela pode ou não ser utilizada para identificar sujeitos com potencial suicida e, então, poder estruturar estratégias de intervenção e prevenção junto a estes.

Não foram encontrados estudos publicados que tenham feito uso da PPAS, exceto o de Lester (2000) e o do próprio Shneidman (1999), que a utilizou apenas com pacientes que estavam em atendimento em seu consultório. Nesses trabalhos não há referência ao tratamento e análise feitos em relação às respostas dos indivíduos ao instrumento, o que possibilitou certo grau de liberdade em sua utilização neste estudo, tanto para administração da PPAS quanto para sua interpretação e manejo dos dados coletados, com a devida autorização do autor.

Para o estudo da fidedignidade, dois métodos foram utilizados. Pelo primeiro, para verificar a consistência interna do instrumento, observou-se que a PPAS possui um coeficiente de fidedignidade de 0,55. Fachel e Camey (2000) sustentam que não existem testes que verifiquem a significância estatística do coeficiente alfa (coeficiente de fidedignidade), entretanto, é usual que o valor 0,70 seja considerado como limite inferior aceitável. Dessa forma, o coeficiente encontrado para a PPAS representa uma possível contribuição de cada uma das questões do instrumento para seu poder, como um todo, de investigar o construto de dor psicológica com confiabilidade e precisão, mas não se tem certeza desta relação.

Encontrou-se em Cunha (2001) a afirmação de que coeficientes de fidedignidade de intensidade leve, como no caso da PPAS, podem estar relacionados aos efeitos do fator de desejabilidade social, que pode ser conceituada como a tendência quase que universal dos indivíduos de escolher uma resposta que é tomada como socialmente mais desejável ou aceitável do que as outras e é, em grande parte, inconsciente (Edwards, 1970). Isso pode se relacionar à necessidade do sujeito de autoproteção, conformidade, aprovação social e evitação das críticas.

Merece igual destaque o fato de que, mesmo tendo se chegado a um coeficiente alfa, em princípio, fraco, todos os itens da PPAS apresentaram uma relação substancial com o total corrigido, em um nível de significância de $p<0,001$. Isso sugere que, mesmo não havendo evidências fortes de relação entre os itens do instrumento no que diz respeito à identificação de dor psicológica, também não há evidências de que não existe 
esta relação; portanto, se conclui que ela é possível. Para dados mais precisos e conclusivos, outros estudos precisam ser realizados, principalmente com populações clínicas e com administração individual do instrumento.

O outro método utilizado na verificação da fidedignidade da PPAS foi o do teste-reteste, entendendo que o instrumento pode ser considerado mais estável como medida no tempo quanto mais próximos forem os resultados obtidos em diferentes momentos de mensuração (Fachel \& Camey, 2000). Encontrou-se uma estimativa de correlação entre teste e reteste da PPAS, com intervalo de 10 dias (Cunha, 2001), de 0,93 ( $p<0,001)$, o que sugere uma correlação satisfatória. Este achado, que parece discrepante do encontrado para o coeficiente de consistência interna, pode ser explicado pelo fato de apenas 39 dos 525 adolescentes da amostra terem realizado o reteste; e mais, acidentalmente, nenhum desses 39 apresentou ideação suicida na BSI. Assim, é possível pensar que a correlação encontrada para estabilidade temporal na PPAS pode ter sido atravessada e influenciada por esses fatores, uma vez que, em um estudo de Lightfoot e Oliver, citado por Cunha (2001), observaram-se dados que sugeriam que sujeitos de população não-clínica e sem comprometimento emocional tendiam a apresentar maior estabilidade de resposta, considerando o tempo transcorrido entre aplicação e reaplicação do instrumento.

A validade de um instrumento investiga se realmente ele mede o que diz estar medindo, e há vários procedimentos para determiná-la. A validade discriminante relaciona-se à validade de construto, e verifica se o instrumento em questão é capaz de discriminar sujeitos que pertencem a diferentes categorias (Fachel \& Camey, 2000), neste caso, os com e os sem dor psicológica.

Buscando justificativas para os achados de que as variáveis de maior influência na avaliação de presença ou não de dor psicológica são "dor psicológica no momento da aplicação", "pior dor psicológica experienciada" e "tentativas anteriores de suicídio", pode-se pensar que estas retratam: a) o momento atual do sujeito, vinculado a eventos recentes geradores de emoções negativas; b) a revivência, através da "pior dor", das necessidades psicológicas frustradas ao longo da vida; e c) a busca concreta de soluções pouco adaptativas para aliviar o sofrimento, representada pelas tentativas de suicídio. Todos esses três aspectos são apontados pela literatura nacional e internacional como preditores do risco de suicídio e, conforme os postulados de Shneidman (1993, 1998, 1999), de presença de dor psicológica.

Também percebeu-se que, para classificar os adolescentes como não tendo ideação suicida na BSI, de acordo com a ausência de dor psicológica, a PPAS mostrou-se muito satisfatória, atingindo 95,5\% de acertos. Para a classificação de adolescentes com ideação suicida na BSI, conforme presença de dor psicológica, o nível de acertos da PPAS é de $42 \%$, tendo como média de acertos $76,4 \%$. Para contemplar este dado, é importante lembrar que a amostra deste estudo foi constituída por adolescentes da população geral. Acredita-se que é por isso que a PPAS discrimina com maior nível de confiança os adolescentes sem ideação suicida na BSI do que os que se enquadram em tal situação.

Quanto à validade convergente, que "verifica se a medida em questão está substancialmente relacionada a outras formas de medida já existentes do mesmo construto" (Fachel \& Camey, 2000, p. 164), e que se relaciona, igualmente, com a validade de construto, comparou-se o escore total da PPAS com o escore total da BSI, da BHS e com o item 9 do BDI. Para todos os três instrumentos de comparação, chegou-se a uma correlação de fraca a moderada, o que significa que o resultado na PPAS independe do resultado em qualquer um dos outros instrumentos (BSI, BHS e item 9 do BDI). Este dado pode ser interpretado se pensarmos que a PPAS foi proposta para que explorações das relações entre dor psicológica elevada e atos suicidas pudessem ser estimadas (Shneidman, 1999), e que não existem outros instrumentos com tal proposta publicados. Logo, no momento em que a validade convergente investiga se há relação entre o instrumento em questão e outros que avaliem o mesmo construto teórico dor psicológica - espera-se que se tenham instrumentos ou itens de comparação. No caso da PPAS, partiu-se de uma premissa ao investigar a correlação entre um instrumento que avalia dor psicológica e outro que avalia ideação suicida (BSI), desesperança (BHS) e idéias suicidas (item 9 do $\mathrm{BDI}$ ): três elementos que são preditores do risco de suicídio. Tal premissa se refere ao fato de que a elevada dor psicológica associa-se à presença de ideação suicida e que sua relação pode ser diretamente proporcional.

No estudo em que Neimeyer e Feixas (em Cunha, 2001) observaram uma correlação baixa entre o BDI e outra escala de depressão, a Escala de Depressão de Hamilton, explicou-se o achado pelo fato de que "as duas medidas não fornecem avaliação idêntica da sintomatologia depressiva" (Cunha, 2001, p. 42). A PPAS e a BSI, a BHS e o item 9 do BDI não avaliam o mesmo construto teórico, logo, correlacionam-se apenas levemente, ou seja, mesmo que todos os instrumentos avaliem fatores preditores do risco de suicídio, e que não haja uma correlação forte entre a PPAS e os demais, isso não a impossibilita de ser um instrumento adequado para a avaliação de dor psicológica.

A respeito da análise fatorial, Fachel e Camey (2000) apontam que esta técnica estatística permite a verificação da unidimensionalidade do construto que está sendo medido, relacionando-se, também, com a validade de construto. Na PPAS, pela análise dos componentes 
principais, chegou-se a três fatores, dos quais apenas o fator II, composto pelos itens "dor psicológica no momento da aplicação" e "pior dor psicológica experienciada", contribui para explicar a presença de dor psicológica, discriminando adolescentes com e sem ideação suicida. Este achado é coerente com os outros resultados obtidos na investigação da validade discriminante, pelo modelo de regressão logística, os quais demonstram que os itens da PPAS que efetivamente influenciam na identificação de adolescentes com e sem dor psicológica são os que compõem o fator II.

O agrupamento das figuras 2, 3 e 4 no fator I parece compreensível, uma vez que as três podem ser facilmente relacionadas à conteúdos negativos e/ou depressivos. Já as figuras 1 e 5, que compõem o fator III, são mais ambíguas para conteúdos de sofrimento. Assim, a análise fatorial permite levantar a suposição de que estes dois fatores ou as cinco figuras podem ajudar a explicar outra dimensão, que não a presença de dor psicológica, fato este que deverá ser melhor investigado.

\section{Conclusões}

Diante de um evento tão trágico e que acarreta tamanha repercussão na sociedade, como o é o suicídio, esforços que contemplem o desenvolvimento de instrumentos capazes de identificar fatores de risco para a autodestruição sempre devem ser implementados, assim como a busca por sua qualificação.

Sabe-se que a importância da investigação de dados como fidedignidade e validade de determinado instrumento psicológico reside na necessidade de aprimorar os instrumentos e técnicas utilizadas para avaliação psicológica, com o objetivo de garantir sua qualidade técnica e ética. Mesmo não tendo sido encontradas, neste estudo, propriedades psicométricas ideais para a PPAS, observou-se que o instrumento pode ser promissor e útil no trabalho com adolescentes, na tentativa de identificar aqueles com maior predisposição para o comportamento autodestrutivo. É certo que este é um estudo preliminar a respeito da utilidade da PPAS para atingir seus objetivos, e que outros estudos precisam ser realizados para que os achados sobre sua qualificação e adequação possam ser novamente verificados, chegando-se, então, a dados mais definitivos e conclusivos. Estudos de fidedignidade entre avaliadores e de validade com populações clínicas estão em curso, assim como pretende-se, também, investigar e esclarecer o papel que as cinco figuras desempenham no instrumento.

\section{Referências}

American Psychiatric Association (2005). Diretrizes para o tratamento de transtornos psiquiátricos. Porto Alegre: Artmed.
Beck, A. T. \& Steer, R. A. (1991). Beck scale for suicide ideation. Manual. San Antonio, TX: Psychological Corporation.

Beck, A. T. \& Steer, R. A. (1993a). Beck depression inventory. Manual. San Antonio, TX: Psychological Corporation.

Beck, A. T. \& Steer, R. A. (1993b). Beck Hopelessness Scale. Manual. San Antonio, TX: Psychological Corporation.

Costa Neto, P. L. O. (1983). Estatística. São Paulo: Blücher.

Cunha, J. A. (2001). Manual da versão em português das Escalas Beck. São Paulo: Casa do Psicólogo.

De Leo, D., Bertolote, J. \& Lester, D. (2003). La violencia autoinfligida. Em E. G. Krug, L. L. Dahlberg, J. A. Mercy, A. B. Zuvi \& P. R. Lozano. Informe mundial de la violencia y de la salud (pp. 200-231). Washington: Organización Panamericana de la Salud.

Edwards, A. L. (1970). The measurement of personality traits by scales and inventories. New York: Holt, Rinehart and Winston Inc.

Fachel, J. M. G. \& Camey, S. (2000). Avaliação psicométrica: a qualidade das medidas e o entendimento dos dados. Em J. A. Cunha. Psicodiagnóstico-V. 5 a ed. (pp. 158-170). Porto Alegre: Artmed.

Leenaars, A. A. \& Lester, D. (1995). Assessment and prediction of suicide risk in adolescents. Em J. K. Zimmerman \& G. M. Asnis (Eds.). Treatment and approaches with suicidal adolescents (pp. 47-70). New York: Wiley-Interscience Publications.

Lester, D. (2000). Psychache, depression and personality. Psychological Reports, 87, 940.

Maris, R. W., Bermann, A. L. \& Silverman, M. M. (2000). Comprehensive textbook of suicidology. New York: The Guildford Press.

Pasquali, L. (1996). Medidas escalares. Em L. Pasquali (Org.). Teoria e métodos de medida em ciências do comportamento (pp. 117-139). Brasília: Laboratório de Pesquisa em Avaliação e Medida, UnB; INEP.

Regulamentação do uso, elaboração e comercialização de Testes Psicológicos, Resolução CFP № 002/2003, Conselho Federal de Psicologia. (2003). Disponível em: <http://www.pol.org.br/resolucoes/ 002_2003.doc>.

Shneidman, E. S. (1993). Suicide as psychache. The Journal of Nervous and Mental Disease, 181 (3), 145-147.

Shneidman, E. S. (1998). Further reflections on suicide and psychache. Suicide and Life-Threatening Behavior, 28(3), 245-250.

Psico-USF, v. 10, n. 1, p. 21-29, jan./jun. 2005 
Shneidman, E. S. (1999). The psychological pain assessment scale. Suicide and life-threatening behavior, 29(4), 287-294.

Souza, E. R., Minayo, M. C. S. \& Malaquias, J. V. (2002). Suicide among young people in selected Brazilian State capitals. Cadernos de Saúde Pública, 18(3), 673-683.

Werlang, B. S. G., Borges, V. R. \& Fensterseifer, L. (2004). Estudo de fidedignidade e validade da Escala de Ideação Suicida de Beck (BSI) em adolescentes. Em B. G. Werlang \& N. J. Botega. Comportamento suicida. Porto Alegre: Artmed.

WHO - World Health Organization (2002). Background. Disponível em: <Who.Int.Mental_Health/ Suicide>.

Recebido em junbo de 2004 Reformulado em abril de 2005 Aprovado em junbo de 2005

Sobre as autoras:

Liza Fensterseifer é psicóloga clínica, mestre em Psicologia Clínica pela Pontifícia Universidade Católica do Rio Grande do Sul e professora assistente do curso de Psicologia da Pontifícia Universidade Católica de Minas Gerais.

Blanca Susana Guevara Werlang é psicóloga clínica, doutora em Ciências Médicas - Saúde Mental - pela Unicamp e professora adjunta do Programa de Pós-Graduação em Psicologia da Faculdade de Psicologia da Pontifícia Universidade Católica do Rio Grande do Sul. 